\title{
Influencia de la edad gestacional y maduración renal postnatal en la cinética de gentamicina*
}

\author{
M.C. Fernando Olavaría U. ${ }^{1}$; Q.F. D. Sc. Humberto Dölz V. ${ }^{2}$; M.C. Sergio Krause H. ${ }^{1}$; \\ M.C. Fernando Hermann H. ${ }^{1}$; M.C. Mónica Broussain B. ${ }^{3}$; M.C. Alicia Ide S. ${ }^{3}$; \\ E.L. Teresa Torres D. ${ }^{3}$

\section{Gentamicin pharmacokinetics in term and preterm newborn infants}

The current scheduje for gentamicin administration to newborn infants, in doses of $2.5 \mathrm{mg} / \mathrm{kg}$ every twelve hours during the first week of life was evaluated in pre-term newborns under 35 weeks of gestational age (GA). Pharmacokinetic studies in steady state condjtions were performed at the third and seventh days of therapy (periods $A$ and $B$ respectively) in nine pre-term (GA 30 to 34 weeks) and ten full-term newborns. Minimal gentamicin blood concentrations at period A were $1.96 \pm 0.32 \mu \mathrm{g} / \mathrm{ml}$ in term newborns and $2.51 \pm 0.48 \mu \mathrm{g} / \mathrm{ml}$ in pre-term infants (p $<0.005$ ) and, at period $\mathrm{B}, 1.49 \pm 0.37 \mu \mathrm{g} / \mathrm{ml}$ and $2.33 \pm 0.34$ respectively $(\mathrm{p}<0.001$ ). Gentamicin excretion showed gaod correlations with gestational age (r; $0.654 ; \mathrm{p}<0.005$ ) and creatinine clearence (r: $0.628 ; \mathrm{p}<0.005$ ). (Key words: gentamicin kinetics, blood levels, pre-term infants, newborn.)

1. Instituto de Pediatría. Facultad de Medicina, Lniversidad Austral de Chile.

2. Instituto de Farmacología, Universidad Austral de Chile.

3. Servicio de Pediatr ía, Hospital Regional de Valdivia.

* Trabajo realizado con el apoyo de la Dirección de Investigación y Desarrollo, Universidad Austral de Chile. Proyectos: RS 85-13 y S 86-36. 
La velocidad de filtración glomerular (VFG) en el recién nacido (RN) es menor que la de] adulto, aun cuando se efectúen las correspondientes correcciones por superficie corporal ${ }^{1}$. Existe, además, una relación directa entre VFG $y$ edad gestacional (EG), de modo que mientras más inmaduro es el producto menor es su $\mathrm{VFG}^{2}$

Por otra parte, la vida extrauterina impone una serie de cambios funcionales al rifión del $\mathrm{RN}$, que determinan un rápido incremento de la VFG, la cual se duplica al cabo de la segunda semana de vida en el RN de término (RNT) y en menor magnitud en el RN de pretérmino (RNPT) ${ }^{3}$.

La gentamicina es un antibiótico del grupo de los aminoglicósidos que se utiliza frecuen. temente en asociación con otros antibióticos en infecciones sospechadas o comprobadas de RN. Su excreción es directamente dependiente de la $V F G^{4}$, por lo cual deberian esperarse variaciones en su farmacocinética con la edad gestacional y tiempo de vida postnatal.

Influyentes agrupaciones médicas de otros pai$\operatorname{ses}^{5}$ recomiendan dosis estándar de gentamicina de $2,5 \mathrm{mg} \times \mathrm{kg}$ de peso, cada $\mathrm{I} 2$ horas, para los $\mathrm{RN}$ de cualquier edad gestacional (EG) durante la primera semana de vida, disminuyendo el intervalo entre dosis a $2,5 \mathrm{mg} \times \mathrm{kg}$ cada 8 horas después del séptimo día de vida, independiente de la edad gestacional del RN.

Considerando los antecedentes expuestos, nos propusimos estudiar la farmacocinética de gentamicina en el estado de equilibrio, en poblaciones de $\mathrm{RN}$ de distinta edad gestacional, durante el curso de la primera semana de tratamiento, con el objetivo de determinar si el esquema mencionado puede condicionar niveles potencialmente tóxicos en los RN de menor edad gestacional.

\section{MATERIAL Y METODO}

Se estudiaron $19 \mathbf{R N}$ de ambos sexos, ingresados a la unidad de neonatologín del Servicio de Pediatría del Hospital Regional de Valdivia, entre el 2 de enero y el 30 de septiembre de 1988 , con antecedente de rotura prematura de membranas y sospecha de infección ovular, sin evidencia de otras afecciones.

De acuerdo a edad gestacional se conformaton dos grupos de pacientes. EI grupo I incluyó 10 RNT (38 a 42 semanas de EG) y el grupo II a 9 RNPT ( 30 a 34 semanas de EG). La EG fue asignada de acuerdo a la historia menstrual y evaluación pediátrica, según el esquema de Dubowitz y Dubowitz ${ }^{6}$.
Todos los RN ingresaron al estudio antes de las 24 horas de vide. Se excluyeron aquellos cuyas madres habían recibido gentamicina u ot to aminoglicósido en las 72 horas previas al parto.

Desde el ingreso, se administró a ambos grupos una dosis de gentamicina de $2,5 \mathrm{mg} \mathrm{x} \mathrm{kg}$ de peso, cada 12 horas, vía i.m., por un período no inferior a 7 días.

Las concentraciones plasmáticas de gentamicina fueron obtenidas al tercero (período A) y séptimo día (período B) del tratamiento. Ambos periodos son representativos de condiciones aparentes de estado de equilibrio de gentamicina en el plașma. El tercer día se elizió por corresponder a un tiempo aproximadamente 5 veces la vida media esperada (Ej.: t $1 / 2=10$ a 15 h) de la fase $\beta$ de eliminación ${ }^{7}$.

De tal manera, se midieron las concentraciones plasmáticas de gentanicina $0,5 \mathrm{~h}$ antes de la sexta dosis i.m. (concentración predosis 1) y media hora después de dicha dosis (concentración postdosis I). Iguales determinaciones se efectuaton antes de la dosis decimocuarta (concentración predosis II) y después de ella (concentración postdosis I). Las muestras obtenjdas fueron congeladas a $-20^{\circ} \mathrm{C}$ y postetiormente analizadas por el método de enzimoinmunoensayo en fase homogénea (EMITQ) ${ }^{6}$.

La constante de velocidad de eliminación $\left(K_{\mathrm{e}}\right)$ se determinó considerando las concentraciones plasmáticas máximas y mínimas de gentamicina en base a la sigujente fórmula 9 :

$$
K_{\mathrm{e}}=\frac{\text { In Cmáx / Cmin }\left(\mathrm{h}^{-1}\right)}{\mathrm{t}}
$$

La vida medja se determinó de acuerdo a:

$$
t 1 / 2=\frac{0,693 \quad(h)}{K_{e}}
$$

Paralelamente a los estudios farmacocinéticos y en los mismos per iados se evaluó función renal mediante medición de aclaramiento de creatinina, para lo cual se recolectó orina de $\mathbf{2 4}$ horas, utilizando recolector autoadhesivo (Terumo $(B)$ durante el tercero y séptimo día de tratamiento. Curnplido el tiempo de recolección de orina, se tomó en ambos periodos una nuestra de sangre venosa. Las determinaciones de creatinina sérica y urinaria fueron hechas mediante técnica de Jaffé.

El análisis estad istico de los resultados fue practicado en un computador digital 2020. Se utilizó la prueba de Student para muestras pareadas e independientes, además de análisis de correlación de Pearson, fijándose la probabilidad de error en menos de $5 \%$.

\section{RESULTADOS}

En las tablas 1 y 2 se comparan los resultados obtenidos para ambos grupos en el periodo A (tercer día de tratamiento) y $B$ (séptimo día de tratamiento) $y$ las diferencias intragrupales en ambos periodos, respectivamente.

Analizando ambas tablas en conjunto, se observa un valor significativamente más alto para el 
Tabla 1

Cinética de gentamicina en RN.

Comparación ambos grupos iguales períodos

\begin{tabular}{|c|c|c|c|c|c|c|c|c|}
\hline \multirow[b]{3}{*}{ Depur. Cr. (ml/mir) } & \multicolumn{5}{|c|}{ PERIODO A } & \multicolumn{3}{|c|}{ PERIODO B } \\
\hline & \multicolumn{3}{|c|}{ RNT } & \multirow{2}{*}{$-\frac{p}{(<0,05)}$} & RNPT & RNT & $\mathrm{p}$ & RNFT \\
\hline & 6,65 & \pm & 5,59 & & $2,16 \pm 1,13$ & $13,53 \pm 8,61$ & $(<0,01)$ & $\pm 2,67$ \\
\hline Conc. Mín. $(\mu \mathrm{g} / \mathrm{ml})$ & 1,96 & \pm & 0,32 & $(<0,05)$ & $2,51 \pm 0,48$ & $1,49 \pm 0,37$ & $(<0,01)$ & $\pm 0,34$ \\
\hline Conc. Máx. $(\mu \mathrm{g} / \mathrm{ml})$ & 4,91 & \pm & 0,67 & $(\mathrm{n} . \mathrm{s})$ & $4,96 \pm 0,43$ & $4,59 \pm 0,59$ & (n.s ) & $5,23 \pm 0,72$ \\
\hline$K_{e}\left(h^{-1}\right)$ & 0,085 & \pm & 0,01 & $(<0,01)$ & $0,064 \pm 0,01$ & $0,105 \pm 0,03$ & $(<0,01)$ & $0,075 \pm 0,01$ \\
\hline $11 / 2$ (b) & 8,30 & \pm & 1.09 & $(<0,05)$ & $11,40 \pm 2,61$ & $6,9 \pm 1,6$ & $(<0,05)$ & $9,50 \pm 1,36$ \\
\hline
\end{tabular}

Depur. $\mathrm{Cr}_{1}=$ Aclaramiento de creatinina. Conc. $=$ Concentración. Mín. $=$ Mínima. Máx. $=$ Máxima. $\mathrm{K}_{\mathrm{e}}=$ Constante de eliminación. $t 1 / 2=$ vida media.

Cafras expresan promedios $(\bar{X}) \pm$ desviación estándar $(D F)$.

Tabla 2

Cinética de gentamicina en RN.

Comparación ambos periodos en igual grupo

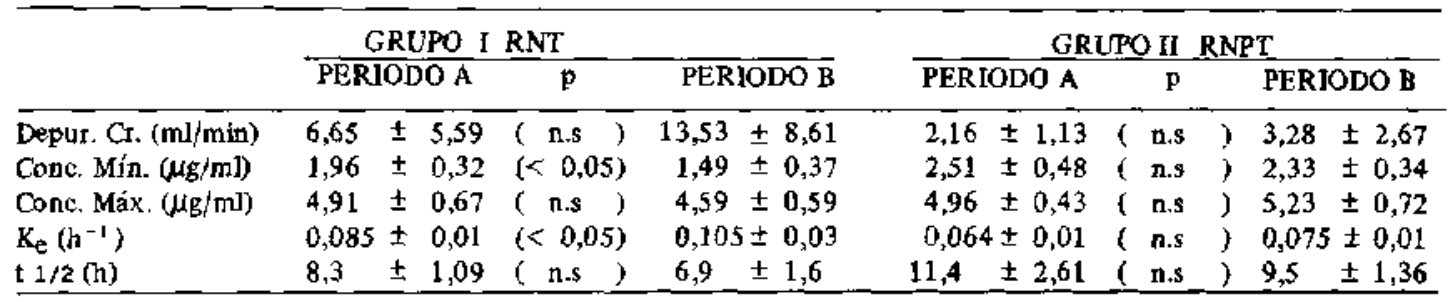

Depur. Cr. = Aclaramiento de creatinina. Conc. $=$ Concentraciōn. Mín. = Mínima. Máx. = Máxima. $\mathrm{K}_{\mathrm{e}}=$ Constante de eliminación. $t 1 / 2=$ vida media.

Cifras expresan promedios $(\overline{\mathrm{X}}) \pm$ desviación estándar (DE).

aclaramiento de creatinina en RNT que en RNPT en ambos períodos.

Los valores de depuración de creatinina observados al séptimo día fueron mayores que los observados al tercer dia para ambos grupos; sin embargo, estas diferencias no alcanzaron significación estadística.

Las concentraciones plasmáticas mínimas de gentamicina en RNT estuvieron, en promedio, por debajo de $2 \mu \mathrm{g} / \mathrm{ml}$ para ambos períodos, no asi en RNPT, en que el promedio estuvo sobre los $2 \mu \mathrm{g} / \mathrm{ml}$ en ambos periodos, diferencias que son significativas en cada uno de los períodos.

Analizados individualmente, sólo 2, de los 10 RNT, tuvieron concentraciones minimas sobre $2 \mu \mathrm{g} / \mathrm{ml}$ en el periodo $\mathrm{A}$, no existiendo ninguno sobre este nivel en el período B. En cambio, se observaron concentraciones mínimas de gentamicina sobre $2 \mu \mathrm{g} / \mathrm{ml}$ en $8 / 9$ RNPT en el período A y en $7 / 9$ durante el período $B$.
Las concentraciones plasmáticas máximas de gentamicina no muestran diferencjas para el mismo grupo en los distintos períados, ni tampoco diferencias significativas al comparar ambos grupos en cada uno de los períodos.

La constante de eliminación $\left(\mathrm{K}_{\mathrm{e}}\right)$ fue significativamente mayor en RNT que en RNPT en ambos períodos. Al comparar los resultados obtenidos para $\mathrm{K}_{\mathbf{e}}$ en el período A y $\mathrm{B}$ se observó un aumento para ambos grupos, pero no alcanza significación estadística.

En la figura 1 se ilustra la relación entre depuración de creatinina y constante de eliminación $\left(\mathrm{K}_{\mathrm{e}}\right)$ en el período $\mathrm{B}$, encontrándose una correla. ción directa y significativa (r: 0,$628 ; \mathrm{p}<0,005$ ).

En la figura 2 se ilustra para igual período la relación entre depuración de creatinina $y$ vida media (t 1/2) de gentamicina, observándose una cortelación inversa y significativa ( $r$ : $-0,567$; p $<0,005)$. 


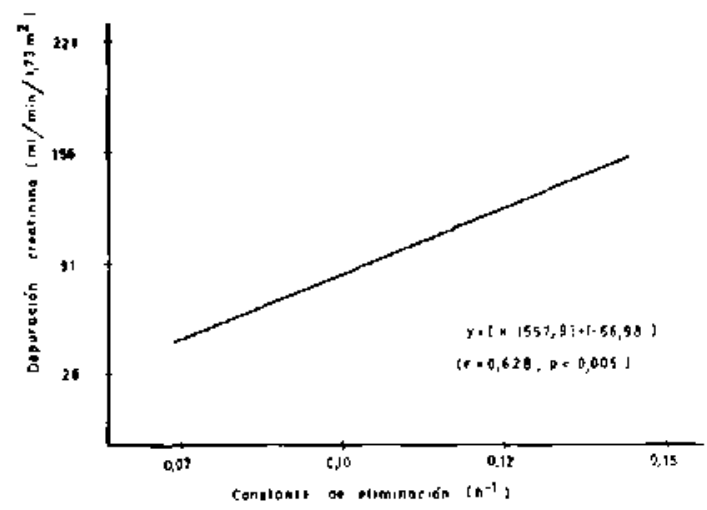

Figura 1: Relación depuración de creatinina y constante de eliminación.

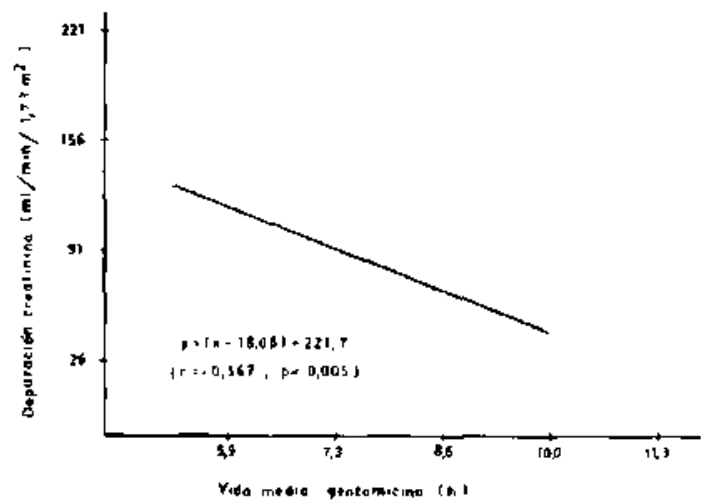

Figura 2: Relación depuración de creatinina y vida media de gentamicina.

Se encontró, además, una correlación directa y significativa entre edad gestacional y $\mathbf{K}_{\mathbf{e}}$ ( $r: 0,654 ; p<0,005$ ) y una correlación inversa $y$ significativa entre edad gestacional y $t 1 / 2$ (s: $-0,654 ; p<0,005$ ).

\section{DISCUSION}

La dosis sugerida de gentamicina de $2,5 \mathrm{mg} / \mathrm{kg}$ cada 12 horas en RN de menos de una semana de vida se ha establecido para lograr concentraciones plasmáticas máximas superiores a $4 \mu \mathrm{g} / \mathrm{ml}$, que estarían sobre la inhibitoria minima para muchos de los gérmenes Gram negativos responsables de sepsis neonatal y meningitis ${ }^{10}$. Por otra parte, $t a l$ esquema terapéutico permite obtener concentraciones plasmáticas mínimas menores de $2 \mu \mathrm{g} / \mathrm{ml}$, sobre el cual se asocia, frecuentemente, mayor riesgo de nefrotoxicidad ${ }^{11}$.
Después de la primera semana de vida se aconseja aumentar la frecuencia de administra. ción de gentamicina a cada 8 horas, basándose en el rápido incremento que experimenta la $\mathrm{VFG}^{10}$ que lleva a un aumento paralelo de depuración de gentamicina $y$, de este modo, acorta su vida media.

De acuerdo a nuestros resultados, la dosis recomendada de gentamicina sería apropiada para RNT; sin embargo, en RNPT el hallazgo de concentraciones mínimas superiores a $2 \mu \mathrm{g} / \mathrm{ml}$ en la mayoría de etlos, observación similar a la descrita previamente por $\mathrm{Killoo}^{9}$, hace conveniente revisar la propiedad de este esquema, especialmente para RNPT de menos de 34 semanas de gestación.

La experiencia descrita por Szeffer ${ }^{12}$, utilizando intervalos mayores de 18 horas en RNPT de menos de 35 semanas, con los cuales logra concentraciones máximas superiores a $4 \mu \mathrm{g} / \mathrm{ml}$ y minimas inferiores a $2 \mu \mathrm{g} / \mathrm{ml}$, señalaría que un incremento en el intervalo entre dosis podría ser Ja solución. Sin embargo, no está suficientemente claro si las concentraciones mínimas superiores a $2 \mathrm{\mu g} / \mathrm{ml}$, potencialmente nefrotóxicas en el adulto, lo sean también para el rinón en desarrollo. Experiencias en RN de muy bajo peso con gentamicina en dosis de $2,5 \mathrm{mg} / \mathrm{kg}$ en intervalos de 12 y 18 horas mostraron en todos ellos concentraciones mínimas superiores a $2 \mu \mathrm{g} / \mathrm{ml}$, sin que esto implicase alteración de la función renal, evaluada a traves de aclaramiento de creatinina y excreción de $\beta 2$ microglobulina ${ }^{13}$. Estos autores explican la ausencia de toxicidad de tales niveles de gentamicina basándose en que a menor edad gestacional predomina el flujo sanguí. neo medular, estando el túbulo proximal cortical en alguna medida protegido. No obstante, se requieren mayores estudios para determinar la validez de tal sugerencia.

La recomendación de acortar los intervalos para dosificación de gentamicina, después de la primera semana de vida, merece también ser comentada. Mientras menor es la edad gestacional, menor es el incremento de VFG observado en la vida extrauterina. En un estudio de RNPT de 28 a 34 semanas se demostró que este cambio no se produce rápidamente en el período postnata ${ }^{14}$, concordando con otras observaciones que demostraron que las diferencias observadas en VFG entre RNPT y RNT se mantienen a lo largo de todo el primer mes de vida ${ }^{3}$.

En apoyo de lo anterior, en un estudio pros- 
pectivo de farmacocinética de gentamicina en RN de muy bajo peso, con dosis estándar de 2 $\mathrm{mg} / \mathrm{kg}$ cada 24 horas, se lograron concentraciones farmacológicas que no variaban sensiblemente durante todo el primer mes de vida ${ }^{9}$.

En nuestra serie, que tiene la limitación de sólo una semana de seguimjento, las concentraciones mínimas de gentanicina permanecieron, al final del período, en valores superiores a 2 $\mu \mathrm{g} / \mathrm{Tl}$ en la mayoría de los RNPT.

En base a los resultados obtenidos y mientras no se definan los niveles potencialmente tóxicos de gentamicina para neonatos de diferente edad gestacional, pareceria razonable utilizar un intervalo mayor entre las dosis para los RNPT de menos de 34 semanas de gestación, manteniendo este esquema más allá de la primera semana de vida. No obstante, dado que no sólo la edad gestacional incide en la maduración, debería practicarse rutinariamente el control individual de concentraciones mínimas y máximas de gentamicina durante el tratamiento en estos recién nacidos.

\section{RESUMEN}

Se evalúa el esquema estándar de gentamicina de $2,5 \mathrm{mg} / \mathrm{kg}$ de peso, cada 12 horas, para recién nacidos durante la primera semana de vida, en una población de recién nacidos de pretérmino de menos de 35 semanas de edad gestacional. Se practicaron estudios de farmacocinética en el estado de equilibrio al tercer y séptimo día (períodos A y B, respectivamente) en nueve recién nacidos pretérmino (edad gestacional 30 a 34 semanas) y se compararon los resultados con un grupo control de diez recién nacidos de término. Las concentraciones mínimas fueron, en el período $A, 1,96 \pm 0,32 \mu \mathrm{g} / \mathrm{dl}$ en RNT $y$ $2,51 \pm 0,48 \mu \mathrm{g} / \mathrm{dl}$ en RNPT $(\mathbf{p}<0,005)$ y, para el periodo $B, 1,49 \pm 0,37$ y $2,33 \pm 0,34$ respectivamente ( $\mathbf{p}<0,001)$. La excreción de gentamicina se correlacionó significativamente con edad gestacional ( $\mathbf{r}: 0,654 ; \mathbf{p}<0,005$ ) y con depuración de creatinina (r: 0,628; p $<0,005$ ). Parece necesario adecuar la dosis de gentamicina para recién nacidos pretérmino de menos de 35 semanas a las limitaciones descritas y conveniente vigilar las concentraciones plasmáticas de este antibiótico.

(Palabras claves: recién nacidos, gentamicina, farmacocinética.)

\section{AGRADECIMIENTOS}

Los autores desean expresar sus agradecimientos al Dr. Luis Barranco C., por su participación en el điseño del proyecto $y$ a la Sra. T.M. Nury Sánchez M., por su colaboración técnica. Así también agtadecen a: Laboratorio Schering Co, por la donación del medicamento Gentalyn ${ }^{\circledR}$ Pediátrico; Laboratorio Merck por proveer los medios para determinar concentraciones de gentamicina plasmática y Laboratorio Clínico Valdivia por las determinacionos bioquímicas efectuadas en este estudio.

\section{REFERENCIAS}

1. Guignard $J$,: Renal function in the newborn infant. Pediatr Clin North Am 1982; 29: 777-790.

2. Otavarria F.. Krause S., Barranco L., Harding $C$. López M.: Función renal en recién zacido de término y pretérnino, durante el segundo dia de vida, Rev Chil Pediatr 1986:57:39-45.

3. Aperia A., Broberger $O$., Elinder $G_{1}$, Hering $P$., Zetterstrom $R$.: Postnatal development of renal function in pre-term and full-term neonates. Acta Pediatr Scand 1981; 70: 183-187.

4. Kaloyanides G., Pastoriza-Muñoz E.: Aminogiycoside nephrotoxicity. Kidney Jnternational. 1980; $18: 571-582$,

5. Red Book, Report of the Committee on Infections Diseases, American Academy of Pediatrics, Twentyfirst edition, 1988.

6. Dubowitz L., Dubowitz V., Golberg C.: Clinical assessment of gestational age in the newborn infart. J Pediatr 1970; 77:1-10.

7. Giacoin G., Gorodisher R. Pharmacologic principles in neonatal drug therapy, Clin Perinatol $1975 ; 2: 125-135$.

8. Kabakoff D., Leung D., Sineh P.: An EMIT ${ }_{\text {assay }}$ for gentamicin. Clin Chem 1978; 24: 1055-1059.

9. Kilóoo C., Mondanlou $H_{-1}$ Komatsu G., Harralson A., Hodding $J_{\text {:: }}$ Developmental pattern of gentamicin kinetics in very low birth weight (VLBW), sick infants. Dev Pharmacol Ther 1984; 7 ; 345-356.

10. McCracken G., Jones L.: Gentamicin in the neonatal period. Amer J Dis Child 1970; 120:524. 533.

11. Schentag $J_{\text {. }}$ Cumbo T., Jusko W., Plent M.: Gentamicin tissue accumulation and nephrotoxic reactions. J Am Med Ass 1978; 240: 2067-2069.

12. Szeffer S., Wynn R., Clarke D., Buckwald Sh., Shen $D .$, Schentag $J$ : Relationship of gentamicin serum concentrations to gestational age in pre-term and term neonates. J Pediatr $1980 ; 97$ (2): 312 . 315.

13. Landers S., Berry P., Kearns G., Kaptan S., Rudolph $A$.: Gentamicin disposition and effect on developmant of renal function in the very low birth weight infant. Dev Pharmacol Ther 1984; $7: 285$ 302 .

14. Arant B.: Developmental patterns of renal functjonal maturation compared in the buman neonate. J Pediatr 1978;92: 705-712. 